\title{
PrICING AMERICAN-STYLE OPTIONS BY SIMULATION
}

\author{
Axel Kind (axel.kind@unisg.ch) \\ Swiss Institute of Banking and Finance, University of St. Gallen, \\ Rosenbergstrasse 52, CH-9000 St. Gallen, Switzerland \\ Tel.: +41-71-2247027, Fax: +41-71-2247088
}

\begin{abstract}
This paper presents an overview of the recent advances in the pricing of American-style securities by simulation. In addition to general considerations related to the use of Monte Carlo simulation in the context of derivatives pricing, five approaches that address the valuation of early-exercisable options are presented: the regression approach, the parametric approach, the stratification approach, simulated trees, and stochastic meshes. The paper provides a brief discussion of each approach with references to the most significant contributions in the academic literature.
\end{abstract}

\section{Introduction}

The valuation of early-exercisable options, or American-style options, is a task of major importance when pricing a number of financial instruments such as equity options, foreign-exchange derivatives, credit and interest-rate derivatives, derivatives on commodities, callable corporate and sovereign bonds, convertible bonds, and mortgagelinked securities. While the economic urge of coping with early-exercisable options is enormous, few pricing techniques can deal in a satisfactory way with this feature. So far, the financial literature has only provided analytical formulae of American-style options for special cases, when early exercise can a priori be ruled out and the American feature is worthless. Although some authors have presented analytical approximations for certain early-exercisable options (e.g., GESKE and JOHNSON (1984) and BARONE-ADESI and WHALEY (1987)), exact closed-form solutions are in general not available for this class of instruments. Therefore, the pricing of American-style securities is typically accomplished my means of numerical methods. Traditionally, two approaches are used for pricing American style options: finite difference methods and binomial trees. Surprisingly, until the mid nineties, Monte Carlo Simulation was explicitly considered not suitable for pricing American-style derivatives. In this currentresearch survey, we review the major arguments and contributions that have led to a change of this paradigm. However, in view of the growing body of literature in the field of numerical option pricing, this article does not claim or attempt to be exhaustive.

\section{Monte Carlo Simulation}

Before we start exposing different approaches for pricing American-style securities, it is useful to focus on some general aspects of simulation-based option pricing. The core of Monte Carlo Simulation is the calculation of an expected value of discounted payoffs, $\widehat{V}_{0}$, over a space of samplepaths, 


$$
\widehat{V}_{0}=\frac{1}{N} \sum_{i=1}^{N} e^{-r \tau_{i}^{*}} h\left(X_{i}, \tau_{i}^{*}\right)
$$

where $N$ is the number of simulation paths, $r$ is the interest rate, $\tau_{i}^{*}$ is the optimal stopping time in path $i, X_{i}$ is the value of the state variable(s) in path $i$, and $h\left(X_{i}, \tau^{*}\right)$ is the payoff generated by the option when exercised at the optimal stopping time. Equation (1) is an approximation of an integral over the risk-neutral density of state variables. The simulation error, i.e., the difference between the Monte Carlo estimate, $\widehat{V}_{0}$, and the true expected value, $V_{0}$, is approximately normally distributed as:

$$
\widehat{V}_{0}-V_{0} \sim N\left(0, \frac{\sigma_{V}}{\sqrt{N}}\right),
$$

where $\sigma_{V}$ is the standard deviation of payoffs and $N$ is the number of simulation paths. Thus, simulation techniques deliver at very low computational cost an estimate of the pricing (in)accuracy. In fact, $\sigma_{V}$ can be estimated as the sample standard deviation of the option payoffs. From equation (2) it is clear that the simulation error decreases with the square root of the number of simulation paths. This means that one decimal point of additional precision requires 100 times as many simulation paths. Although this error convergence rate is rather slow if compared to alternative pricing techniques, pricing options by simulation offers a number of decisive advantages:

First, the convergence rate of the simulation approach is mostly independent of the number of state variables, which makes Monte Carlo Simulation computationally appealing for pricing problems with multiple sources of risk, if compared to conventional binomial trees or finite difference methods. Second, Monte Carlo Simulation is highly flexible with respect to the evolution of the state variables. Instead of relying on conventional processes such as geometric Brownian motions, simulation techniques offer the opportunity to price derivatives with more complex and realistic process dynamics.[1] Third, while some path-depen- dent option features can hardly be priced by traditional lattice methods, they can easily be included in a Monte Carlo pricing framework. Fourth, intermediate cash flows such as coupon payments and dividends occur discretely in time and can much easier be modeled. Finally, Monte Carlo Simulation is a general, intuitive, and versatile pricing method, which makes it particularly appealing for practical purposes.

\section{Simulation Approaches}

In this section, we briefly present and discuss the most important approaches for pricing American derivatives by simulation.[2] To facilitate the exposition we sometimes refer to standard put options as a simple, yet representative, example of American-style security. In fact, the majority of articles on numerical option pricing employ standard put and call options to relate the performance of new models to existing ones.

\subsection{The Regression Approach}

In many cases of interest, it is straightforward to express the conditions under which an Americanstyle option is exercised in terms of the relationship between the value from immediate exercise and the continuation value, i.e., the value of the option given it is not immediately exercised. Clearly, it is optimal to exercise the option only if the continuation value is lower than the value of immediate exercise. The regression approach aims at modeling the early-exercise feature by finding, at each point in time and for any value of the state variables, the correct value of continuation of the security under scrutiny. This goal is achieved by exploiting the information contained in the cross section of simulated sample paths, while working backwards from maturity to time zero. More precisely, the core of the approach consists of regressing, at each point in time, the cross section of future 
discounted cash flows on a number of basis functions (e.g., polynomials) of the state variables. The systematic relationship obtained from this regression is called conditional-expectation or continuation-value function. It enables one to determine the continuation value of an option given a certain value of the state variables. CARRIERE (1996) proposes the use of non-parametric regression techniques for pricing American call and put options. LONGSTAFF and SCHWARTZ (2001) show how a least-squares regression technique can be used for pricing a range of financial instruments including American put options (with and without jumps), American-Bermuda-Asian options, cancelable index amortizing swaps, and swaptions. KIND and WILDE (2003) use a similar least-square regression method to price convertible bonds with multiple embedded options and additional pathdependent features. TSITSIKLIS and VAN ROY (1999, 2001) stress the close relationship between the American option pricing task and problems in the field of neuro-dynamic programming. They further provide a technical treatment of the regression-based pricing approach and present convergence proofs.

\subsection{The Parametric Approach}

The main idea of the parametric approach is to represent the exercise behavior of the investor as a parametric function of state variables (e.g., the stock price) and time. The complexity of the pricing problem is reduced by choosing the best exercise behavior among a specific class of functions. The parameterization of the exercise behavior can either be obtained by a parametric class of stopping times or by a parametric class of exercise regions. Since these two parameterizations are in principle equivalent, we can focus on the latter for a brief exposition of the methodology. The space of all possible values of state variables is split into several disjoint regions, each related to a different behavior of option holder. For the simple case of an American put option with constant interest rates, at each point in time, two regions for the underlying stock price are defined: one where the option is exercised and one where it is not. Over time, these two regions can be separated by a so called exercise boundary. When the stock price falls below the exercise boundary, the put option is exercised. Thus, the exercise behavior of the investor is fully described by the exercise boundary. Since the optimal exercise boundary is unknown, it is critical to allow for a wide range of possible exercise policies. The parametric approach achieves this by defining a flexible class of parametric functions. For instance, GARCIA (2003) presents numerical results obtained by connecting three values of the exercise boundary, for three different maturities, through cubic-spline interpolation. JU (1998) approximates the exercise boundary by a piecewise exponential function. Most authors agree that even a simple approximation of the exercise region generates fairly accurate pricing results, as the sensitivity of option prices on the shape of the exercise boundary is not very high. Knowledge about the optimal exercise policy derived from option theory can be included in the pricing approach by restricting the shape of the exercise boundary. For instance, it is known that an American put option is exercised at maturity whenever the stock price falls below the strike price. The aim of the parametric pricing approach is to find the optimum exercise strategy, which generates the highest value of the option, by numerically maximizing over a parameter space. For this reason, the parametric approach is often referred to as an optimization approach. Besides finding the price of the option, the parametric approach delivers an exercise rule valuable for the investor. It is worth noting that the parametric approach is the only one that does not require backward induction: The option value is determined by simulating forward path wise until either the state variables cross the exercise boundary and fall into the exercise region or maturity is reached. BOSSAERTS (1989) was the first to propose a 
parametric approach to price American options. Since then, several other authors have refined and expanded this technique to price a wide range of derivative securities. GRANT et al. (1997) apply a version of the parametric approach to value American-style Asian options. ANDERSEN (2000) uses a parametric approach for pricing Bermudan swaptions. AMMANN et al. (2005) use a parametric technique to perform an empirical pricing study on convertible bonds. GARCIA (2003) focuses on potential pricing biases and presents numerical examples for American call options and options on the maximum of two and five assets, often called rainbow options.

\subsection{The Stratification Approach}

Similarly to the regression approach presented above, the stratification approach simulates paths for the state variables and applies a backwardinduction procedure for determining the optimal exercise behavior in each node. However, the conditional expectation function is computed by means of a stratification of the sample space. The different contributions mainly differ with respect to the ways this stratification is performed and the associated dynamic programming algorithm is solved. Examples of these approaches applied to standard American options and options on the maximum of multiple assets are provided by TILLEY (1993), BARRAQUAND and MARTINEAU (1995), and RAYMAR and ZWECHER (1997)

\subsection{The Simulated Tree Approach}

The graphical representation of a simulated tree, or random tree, strongly resembles a non-recombining multinomial tree: $b$ branches depart from an initial node with the current value of the state variable. Each of these branches represents a distinct change of the state variable. From each of these $b$ branches, again $b$ new branches are simulated. This compounded simulation procedure is repeat- ed $n$ times until maturity. However, unlike nonrecombining trees, the branches of simulated trees are sampled randomly from an appropriate conditional density and are not determined by a deterministic rule. The price of the put option is determined via backward induction. At maturity, the payoff of the put option in $\operatorname{Max}\left(K-S_{T}, 0\right)$, where $K$ is the strike price and $S_{T}$ is the stock price at maturity. In each node before maturity, the decision whether to exercise or not is based on the relationship between the continuation value and the value from immediate exercise. The continuation value is determined as the average of the payoffs generated by the option in the following $b$ nodes. While the described algorithm produces an upward-biased estimate of the true option value, a downward-biased variant can be achieved as well. Due to the extensive branching, computational costs grow exponentially with the number of exercise dates, making this approach only suitable for pricing problems with up to five exercise dates. Nevertheless, BROADIE and GLASSERMAN (1997), the proponents of this approach, stress the two positive features of this pricing technique. First, random trees do not rely on approximations of the early exercise strategy or conditional expectation functions. Second, the method allows, through appropriately tailored backward induction algorithms, the generation of two option price estimates, one biased low and one biased high, both converging to the true option value as $b$ tends to infinity. By combining these two estimates it is possible to build a conservative confidence interval very similar to one based on a truly unbiased estimator. Building on the basic algorithm for simulated trees, BROADIE et al. (1997a, b) propose a series of enhancements to improve the efficiency of this pricing technique.

\subsection{The Stochastic Mesh Approach}

The stochastic mesh approach developed in BROADIE and GLASSERMAN (2004) and 
BROADIE et al. (1997a, b) is a very general derivative pricing technique. For instance, GLASSERMAN (2004) shows that stochastic mesh methods nest the regression method of LONGSTAFF and SCHWARTZ (2001). A stochastic mesh can be obtained by simulating path wise the relevant state variables until maturity (independent-path construction). However, when moving backwards from maturity, the continuation value is calculated as weighted average of the payoffs occurring in all nodes of the following time step. As noted by GLASSERMAN (2004), it is precisely the choice of the weights used for relating each node to the next ones that crucially differentiates the various versions of stochastic meshes. It is worth noticing that in each node the branching resembles a simulated tree. However, unlike simulated trees, the number of nodes at each point in time is constant, which is computationally appealing.

\section{Additional Issues}

\subsection{Speed and Storage}

In the previous section, all the proposed pricing approaches are presented in form of crude Monte Carlo Simulation, i.e., without addressing the possibility of reducing the variance of the estimate through appropriate techniques. However, given the computational burden of any pricing solution based on simulations, the practical success of a valuation technique critically depends on the ability to achieve a certain pricing precision within an acceptable time span. For instance, a trader might be willing to adopt pricing tools based on simulation, only if the time to price the instrument is in the order of magnitude of a couple of seconds. Since a discussion of variance reduction techniques is beyond the scope of this article, the reader might refer to BOYLE et al. (1997) for a survey on this topic. Further possibilities to speed up the American option pricing algorithms deal with the efficiency of the implementation. For instance, BROADIE et al. (1997a, b), propose several enhancements to increase the pricing efficiency of simulated trees. AVRAMIDIS et al. (2002) investigate the possibility of improvements in speed when pricing American options with a stochastic mesh in a parallel environment. They report nearly perfect efficiency, meaning that computation time decreases almost linearly with the number of processors. While computational costs in terms of time are practically more important than storage requirements, some attention is also given to the latter topic. In fact, storage requirements for pricing American options are fairly high because calculating the continuation value often requires information contained in the cross-section of sample paths. A comparative study on computational costs of different numerical techniques for pricing American options is performed in FU et al. (2001).

\subsection{Convergence and Biases}

Prices generated by Monte Carlo Simulation can suffer from different sources of biases. High biases can arise for example from using future information for determining optimal exercise decisions or, as discussed in GARCIA (2003), by the in-sample use of the optimal exercise boundary. Since exercise rules are optimized to achieve maximum prices on a given set of randomly generated state variables, the optimal exercise strategy will "exploit" the random characteristics of the simulated paths used, especially when the number of paths is small. Low biases arise from inaccurately specified or estimated exercise rules. As of today, several papers have addressed these pricing biases for standard American options, but the magnitude of these biases for more complex American-style derivatives is still an open issue future research will have to address. The duality approach proposed by HAUGH and KOGAN (2004) and ROGERS (2002) reformulates the 
American pricing task in form of a minimization problem over a class of martingales or supermartingales and can be used to obtain suitable and surprisingly tight upper bounds for option prices. ANDERSEN and BROADIE (2004) apply this technique and are successful in finding a tight upper bound for the least-squares regression approach of LONGSTAFF and SCHWARTZ (2001). Finally, CLEMENT et al. (2002) prove the convergence of the least-squares approach by LONGSTAFF and SCHWARTZ (2001).

\section{Summary and Outlook}

This current research article provides an overview of the most recent advances in simulationbased option pricing. This field of research is surprisingly new as the very first contributions date back to the early nineties. Nevertheless, a number of valuable approaches has been proposed. They address important issues such as pricing biases, confidence intervals for pricing errors, computational costs, and storage requirements. Future research will likely concentrate on further enhancements of existing approaches, on the comparison of existing methods, and, most importantly, on empirical studies employing these techniques for both pricing and hedging. Overall we can state that, due to its flexibility, Monte Carlo Simulation is the most valuable tool for coping with the new pricing challenges arising from the increasingly innovative structure of financial securities.

\section{ENDNOTES}

[1] KLOEDEN and PLATEN (1992) discuss a variety of methods for constructing accurate discretetime approximations of stochastic differential equations that may be used for modeling the dynamics of the state variables.

[2] In general, simulation techniques only allow for a finite number of early-exercise times. Hence, these techniques price Bermudan options rather than continuously exercisable American options. However, for a fairly large number of earlyexercise dates, the Bermudan price may serve as an approximation for the price of the American option. 


\section{REFERENCES}

AMMANN, M., KIND A. and C. WILDE (2005): "Simulation-Based Pricing of Convertible Bonds", Working Paper.

ANDERSEN, L. (2000): "A Simple Approach to the Pricing of Bermudan Swaptions in the Multifactor LIBOR Market Model", Journal of Computational Finance 3, pp. 5-32.

ANDERSEN, L. and M. BROADIE (2004): "A Primal Dual Simulation Algorithm for Pricing Multi-Dimensional American Options", Management Science 50, pp. 1222-1234.

AVRAMIDIS, A. N., T. F. COLEMAN, A. VERMA and Y. ZINCHENKO (2002): "Efficiency Improvements for Pricing American Options with a Stochastic Mesh: Parallel Implementation", Working Paper.

BARONE-ADESI, G. and R. E. WHALEY (1987): "Efficient Analytic Approximation of American Option Values", Journal of Finance 42, pp. 301-320.

BARRAQUAND, J. (1995): "Numerical Valuation of High Dimensional Multivariate European Securities", Management Science 41, pp. 1882-1890.

BARRAQUAND, J. and D. MARTINEAU (1995): "Numerical Valuation of High Dimensional Multivariate American Securities", Journal of Financial and Quantitative Analysis 30, pp. 383-405.

BOSSAERTS, P. (1989): "Simulation Estimators of Optimal Early Exercise”, Working Paper, Carnegie Mellon.

BOYLE, P., M. BROADIE and P. GLASSERMAN (1997): "Monte Carlo Methods for Security Pricing", Journal of Economic Dynamics and Control 21, pp. 1267-1321.

BROADIE, M. and P. GLASSERMAN (1997): "Pricing American-Style Securities by Simulation", Journal of Economic Dynamics and Control 21, pp. 1323-1352.

BROADIE, M. and P. GLASSERMAN (2004): "A Stochastic Mesh Method for Pricing High-Dimensional American Options", Journal of Computational Science 7, pp. 35-72.
BROADIE, M., P. GLASSERMAN and G. JAIN (1997): "Enhanced Monte Carlo Estimates for American Option Prices", Journal of Derivatives 5, pp. 25-44.

BROADIE, M., P. GLASSERMAN and Z. HA (1997): "Pricing American Options by Simulation Using a Stochastic Mesh with Optimized Weights", in: Uryasev, S. (ed.), Probabilistic Constrained Optimization: Methodology and Applications. Kluwer Academic Publishers, Norwell, Mass.

CARRIERE, J. F. (1996): "Valuation of Early-Exercise Price of Options Using Simulations and Nonparametric Regression", Insurance: Mathematics and Economics 19, pp. 19-30.

CLEMENT, E., D. LAMBERTON and P. PROTTER (2002): "An Analysis of a Least Squares Regression Method for American Option Pricing", Finance and Stochastics 6, pp. 449-471.

FU, M. C., S. B. LAPRISE, D. B. MADAN, Y. SU and R. WU (2001): "Pricing American Options: A Comparison of Monte Carlo Simulation Approaches", Journal of Computational Finance 4, pp. 39-88.

GARCIA, D. (2003): "Convergence and Biases of Monte Carlo Estimates of American Option Prices Using a Parametric Exercise Rule", Journal of Economic Dynamics and Control 27, pp. 1855-1879.

GESKE, R. and H. E. JOHNSON (1984): "The American Put Option Valued Analytically", Journal of Finance 39, pp. 1511-1524.

GLASSERMAN, P. (2004): Monte Carlo Methods in Financial Engineering, New York: Springer-Verlag. GRANT, D., G. VORA and D. WEEKS (1997): "PathDependant Options: Extending the Monte Carlo Simulation Approach", Management Science 43, pp. 1589-1602.

HAUGH, M. and L. KOGAN (2004): "Pricing American Options: A Duality Approach", Operations Research 52, pp. 258-270.

JU, N. (1998): "Pricing an American Option by Approximating Its Early Exercise Boundary as a Multipiece Exponential Function", Review of Financial Studies 11, pp. 627-647. 
KIND, A. and C. WILDE (2003): "Pricing Convertible Bonds with Monte Carlo Simulation", Working Paper.

KLOEDEN, P. and E. PLATEN (1992): Numerical Solution for Stochastic Differential Equations, New York: Springer-Verlag.

LONGSTAFF, F. A. and E. S. SCHWARTZ (2001): "Valuing American Options by Simulation: Simple Least-Squares Approach", Review of Financial Studies 14, pp. 113-147.

RAYMAR, S. and M. ZWECHER (1997): "Monte Carlo Valuation of American Call Options on the Maximum of Several Stocks", Journal of Derivatives 5, pp. 7-20.

ROGERS, L. C. (2002): "Monte Carlo Valuation of American Options", Mathematical Finance 12, pp. 271-286.

TILLEY, J. A. (1993): "Valuing American Options in a Path Simulation Model", Transactions of the Society of Actuaries 45, pp. 83-104.

TSITSIKLIS, J. and B. VAN ROY (1999): "Optimal Stopping of Markov Processes: Hilbert Space Theory, Approximation Algorithms, and an Appli- cation to Pricing High Dimensional Financial Derivatives", IEEE Transactions on Automatic Control 44, pp. 1840-1851.

TSITSIKLIS, J. and B. VAN ROY (2001): "Regression Methods for Pricing Complex American-Style Options", IEEE Transactions on Neural Networks 12, pp. 694-703.

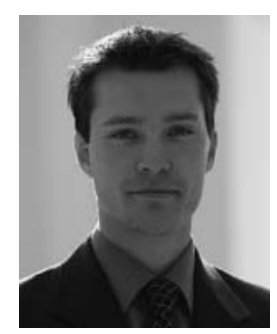

Axel Kind is a Lecturer of Finance at the University of St. Gallen(HSG), Switzerland. Prior to his current appointment he was Research Scholar at the Gradute School of Business of Columbia University and the Computer Science Department of the University of California, Los Angeles. Axel Kind holds a Doctorate Degree in Finance from the University of St. Gallen. His research mainly focuses on topics related to the pricing of derivatives with numerical techniques, convertible securities, endogenous correlations of asset returns, and consumption-based asset pricing. 